\title{
Steady-State Measurements of Respiration Rate with an Oxygen Electrode
}

\author{
J. D. TJepkema And C. S. Yocum ${ }^{1}$ \\ Department of Botany, University of Michigan, Ann Arbor, Michigan 48104
}

Received March 15, 1974; accepted September 6, 1974

\begin{abstract}
An oxygen electrode was developed which measures steady-state respiration rates in a volume of $0.25 \mathrm{ml}$ and at oxygen concentrations as low as $0.1 \mu \mathrm{M}$. The steady state was achieved by pumping air-equilibrated buffer into the respirometer at various rates. The method is most suitable for tissue slices.
\end{abstract}

Respirometers for measuring respiration rates at steady state have been previously described (1), and more recently a method has been published for such measurements at low $\mathrm{pO}_{2}(2)$. We have developed an alternative method for low $\mathrm{pO}_{2}$ that has some advantages, as will be discussed. Examples of the application of the method are given in Tjepkema (3).

\section{METHODS}

The respirometer is shown in Fig. 1. The cathode was made from gold wire of $0.51 \mathrm{~mm}$ diam which was coated with glass of low melting point (4). After mounting the cathode as shown in Fig. 1, the gold and its glass coating were ground to a flat surface and polished using a series of carborundum grinding powders. The cathode was maintained at $-0.45 \mathrm{~V}$ with respect to the calomel electrode. The electrode current was measured with a Keithley model $150 \mathrm{~A}$ microvolt-amperometer (Keithley Instruments, Cleveland, $\mathrm{OH} 44139$ ). The buffer was $0.01 \mathrm{M}$ succinate at $\mathrm{pH} 6.0$, made with glass-distilled water. At low oxygen tensions, $0.01 \mathrm{M}$ $\mathrm{KCl}$ was used as an electrolyte, but at atmospheric oxygen tension, 0.1 $\mathrm{M} \mathrm{KCl}$ was required to prevent appreciable electrolyte resistance between the electrodes. Stirring was done with a magnet rotated at 2000 rpm under the respirometer. The slot along the inner joint of the respirometer was just large enough to accommodate a 26-gauge hypodermic needle. This needle was connected to a syringe pump, and airequilibrated buffer was pumped into the respirometer at a constant rate. The syringe pump was constructed by attaching the chart drive of a Health/Schlumberger model EU-20B recorder (Health/Schlumberger Instruments, Benton Harbor, MI 49022) to the rack and pinion gear

${ }^{1}$ To whom requests for reprints should be addressed. 


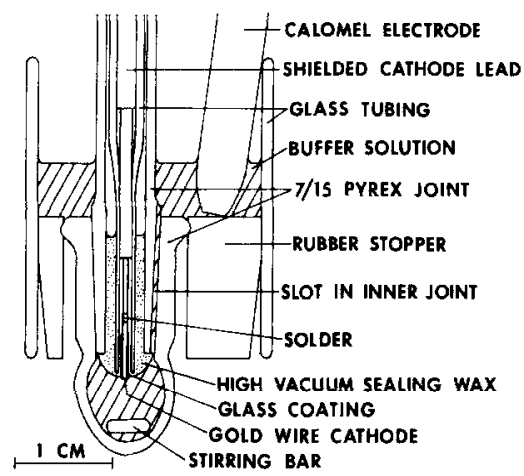

FIG. 1. Oxygen electrode respirometer. Internal volume: $0.25 \mathrm{ml}$.

drive of a microscope body. A $1 \mathrm{ml}$ disposable syringe was used and the pumping speed was calibrated by measuring the length of time required to pump a given volume of water. The calibrations at different chart speeds on the recorder, or at a fixed speed, agreed with each other to within $1 \%$. Starting with anaerobic conditions, 1-2 min were required for the $\mathrm{pO}_{2}$ to increase to a steady state, where the respiration rate equaled the net rate of oxygen flow into the respirometer. The net rate was the difference between the rates at which oxygen entered and left the respirometer. Various oxygen tensions and respiration rates were obtained by varying the pumping rate.

When all oxygen was depleted from the respirometer, a substantial residual current remained. This current was about $5 \mathrm{nA}$ immediately

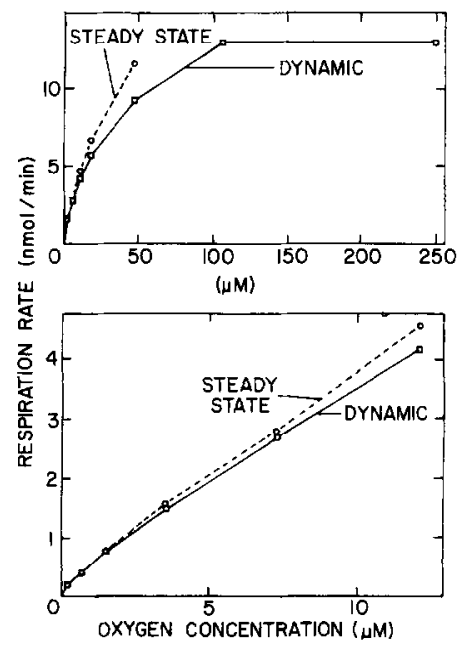

FIG. 2. Respiration of soybean nodule slices as measured by steady-state and dynamic methods. Fresh weight of slices: $4.7 \mathrm{mg}$. 
after oxygen depletion, and then decreased to a steady value of about $0.1 \mathrm{nA}$ after 15-30 min. The residual current fell much less rapidly if there were no tissue slices (from soybean nodules) in the respirometer. The cause of the residual current may have been reducible compounds that contaminated the glassware surfaces and buffer. It was not due to leakage of oxygen through the slot in the inner joint, since the current was not affected by the $\mathrm{pO}_{2}$ of the buffer above the slot. When the residual current was subtracted, the electrode current was directly proportional to the oxygen concentration and was about $1.0 \mathrm{nA} / \mu \mathrm{M} \mathrm{O} \mathrm{O}_{2}$. Air saturated buffer was about $250 \mu \mathrm{M} \mathrm{O}_{2}$.

\section{RESULTS AND DISCUSSION}

Figure 2 shows the results obtained with the respirometer. Soybean nodule slices of $100 \mu \mathrm{m}$ or less thickness were used. This thickness corresponded to one or two cell layers. At each oxygen concentration, the respiration rate was measured by both steady-state and dynamic methods. The steady-state rate was measured first, by setting the syringe pump at a constant rate and then measuring the oxygen electrode current after a steady state was reached. In calculating the respiration rate, correction was made for the oxygen being forced out of the respirometer as the air-equilibrated buffer was pumped in. The oxygen electrode current gave the oxygen concentration corresponding to the calculated respiration rate. The dynamic respiration rate at the given oxygen concentration was measured by increasing the oxygen concentration slightly by increasing the pumping rate, and then completely stopping the pumping. The respiration rate was calculated from the rate of decrease in oxygen concentration at the desired oxygen concentration and from the internal volume of the respirometer.

The results represent a progressive oxygen saturation of the respiratory sites from the outside to the inside of the tissue slices as the oxygen concentration is increased. When the slices were homogenized, a much lower oxygen concentration was required to saturate their respiration. The reason for the disagreement between the steady-state and dynamic methods at high oxygen concentrations is not known.

In view of the above disagreement, there may be inaccuracies in use of this method at high $\mathrm{pO}_{2}$ values. The need for $0.1 \mathrm{M} \mathrm{KCl}$ may also limit use of the method at high $\mathrm{pO}_{2}$, although this need could be eliminated by using a much smaller electrode, so that the current flow through the buffer would be much reduced. Another limitation of the method is that it may not be suitable for very fragile material that might disintegrate upon contact with the stirring bar. Also, if the respiring material is of small size, it will be gradually washed out of the respirometer as buffer is pumped in. This problem is minimized at low $\mathrm{pO}_{2}$, 
because of the much lower pumping rate due to the low respiration rate. The uncoated gold cathode might be unstable in certain experimental systems, but has been stable for our conditions. The electrode current in air-equilibrated buffer varied by about $10 \%$ from day to day. It is important to periodically sterilize the respirometer, since otherwise substantial respiration can be observed in the absence of any added respiring material. In a sterile respirometer, no oxygen consumption was observed. This indicated that oxygen consumption by the electrode was negligible, as was confirmed by calculation.

The advantages of the respirometer are its small volume, ease of construction, ability to be used at low $\mathrm{pO}_{2}$ values, and the ability to rapidly change the composition of the buffer in which tissue slices are suspended. This is done by injecting the desired buffer through the slot in the inner joint of the respirometer.

\section{ACKNOWLEDGMENT}

Professor Jerome Schultz, Department of Chemical Engineering, University of Michigan, was responsible for the suggestion of using a syringe pump for measuring steadystate rates of respiration.

\section{REFERENCES}

1. Larrabee, M. G. (1958) J. Neurochem. 2, 81-101.

2. Degn, H., and Wohlrab, H. (1971) Biochim. Biophys. Acta 245, 347-355.

3. Tjepkema, J. D. (1971) Doct. dissert., University of Michigan. Ann Arbor, Mich.

4. Wolbarsht, M. L., MacNichol, E. F., Wagner, H. G. (1960) Science 132, 1309-1310. 